\title{
Experimental technique to analyze the influence of cutting conditions on specific energy consumption during abrasive metal cutting with thin dises
}

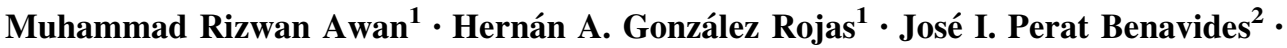 \\ Saqib Hameed ${ }^{1}$
}

Received: 13 January 2021/Revised: 23 March 2021/Accepted: 9 June 2021 / Published online: 9 August 2021

(C) The Author(s) 2021, corrected publication 2021

\begin{abstract}
Specific energy consumption is an important indicator for a better understanding of the machinability of materials. The present study aims to estimate the specific energy consumption for abrasive metal cutting with ultrathin discs at comparatively low and medium feed rates. Using an experimental technique, the cutting power was measured at four predefined feed rates for S235JR, intermetallic $\mathrm{Fe}-\mathrm{Al}(40 \%)$, and $\mathrm{C} 45 \mathrm{~K}$ with different thermal treatments. The variation in the specific energy consumption with the material removal rate was analyzed through an empirical model, which enabled us to distinguish three phenomena of energy dissipation during material removal. The thermal treatment and mechanical properties of materials have a significant impact on the energy consumption pattern, its corresponding components, and cutting power. Ductile materials consume more specific cutting energy than brittle materials. The specific cutting energy is the minimum energy required to remove the material, and plowing energy is found to be the most significant phenomenon of energy dissipation.
\end{abstract}

Keywords Specific cutting energy - Specific energy consumption - Metal cutting with abrasive disc - Abrasive cut off operation - Cutting intermetallic alloy $\mathrm{Fe}-\mathrm{Al}(40 \%)$

Muhammad Rizwan Awan mrawan@kth.se

1 Department of Mechanical Engineering, Universitat Politècnica de Catalunya (UPC), Barcelona, Spain

2 Department of Electrical Engineering, Universitat Politècnica de Catalunya (UPC), Barcelona, Spain

\section{Introduction}

The industrial sector is currently responsible for $85 \%$ of the world's energy, with manufacturing accounting for $40 \%$ of the energy used in the industry [1]. To minimize the environmental impact and reduce the production costs, a reduction of the energy consumption is among the greatest challenges for the manufacturing industry [2,3]. Machining is one of the most important manufacturing technologies and represents a major energy demand [4]. To reduce its energy consumption, an understanding and estimation of the energy consumed are the primary and important steps [5]. The specific energy consumption, that is, the energy required to remove the unit volume of a material, is an important metric for evaluating the energy consumption of machining [6]. In terms of the specific energy consumption, grinding is one of the most energy-intensive processes among other metal cutting operations. During grinding, the largest portion of the emissions is attributed to the energy used for material removal [7]. The grinding process, which emphasizes a high material removal, is an abrasive cut off operation, and is the sectioning of materials with thin cutting discs made of abrasive particles [8,9]. Therefore, a specific energy analysis of the grinding process involving a high material removal is extremely important.

The specific energy consumption from grinding has three components: chip formation energy, plowing energy from the material deformation, and sliding or rubbing energy [10]. Rubbing or sliding is the first stage of grain interaction with the workpiece, and the elastic deformation occurs during this phase with a negligible amount of material removal. With the increase in force, grain penetration into the workpiece also increases, leading to the plowing phase, and both elastic and plastic deformations occur during this phase. Further penetration of grain leads 
to the cutting phase, where the material is plastically deformed to produce chips $[11,12]$. In this paper, the notations $P_{\mathrm{sl}}, P_{\mathrm{pl}}$, and $P_{\mathrm{ch}}$ are used to refer to the power consumed by the three mechanisms mentioned above, that is, sliding, plowing, and cutting energy, respectively.

Various factors such as the abrasive sharpness, material properties, and process parameters govern the transition of energy consumption behavior among these three stages and have been extensively discussed. According to Rowe [13], rubbing or sliding is the predominant form of energy in material removal for processes such as polishing and lapping, whereas precision grinding occurs in all three regimes. High-energy deep grinding and cut-off operations take place predominantly in the material removal regime through a chip formation for ductile materials or through a crack formation for brittle materials. Nápoles et al. [14] developed a material removal rate model to evaluate the specific energy consumption during industrial-scale grinding of $\mathrm{C} 45 \mathrm{~K}$ with different thermal treatments and AISI 304. It was demonstrated that the sliding energy was the main form of energy dissipation and decreased with an increase in the depth of the cut. Masoumi et al. [15] investigated the material removal behavior during surface grinding of a high-velocity oxy-fuel (HVOF) thermally sprayed WC-10Co4Cr coating. The results revealed that material removal was due to both a brittle fracture and ductile flow modes depending on the grinding parameter variation, and a large amount of energy was dissipated by a plastic deformation from the plowing. Similarly, Singh et al. [16] found a specific plowing energy to be the most significant component at lower feed rates for the grinding of hard and high-strength materials. Shaw [17] demonstrated that little energy was carried by the chips when conducting finish and form grinding (FFG), whereas with stock removal grinding (SRG), most of the specific energy was consumed as the shear cutting energy to produce chips. According to Malkin and Joseph [18], at extremely high material removal rates, the sliding and plowing energy became negligible, and the minimum energy was equal to the specific chip formation energy. The minimum energy required to form the chips is constant, and at higher removal rates, it is the only dominant energy [6].

Contrary to many different studies on the specific energy for FFG, the specific energy consumption of an abrasive cut off operation with thin cutting discs for metals has rarely been discussed. In an earlier study, Shaw et al. [19] analyzed the influences of the feed rate on specific energy consumption and cutting forces in an abrasive cut off operation for steel and aluminum. They used a cutting disc with a large diameter $(508 \mathrm{~mm})$ and an extremely high feed rates $(3.39-12.7 \mathrm{~mm} / \mathrm{s})$ for the experiment. The results revealed that the cutting forces increased with an increase in the feed rate up to some extent and then decreased from the trend line. The specific energy consumption decreased with an increase in the feed rate, however, this study did not explain the three specific energy components of rubbing, plowing, and cutting during material removal. Turchetta [20] investigated the cutting conditions that affected the cutting force and cutting energy in stone cutting using diamond cutting disks. The author showed that the cutting energy decreased with an increase in the depth of the cut and the feed rate, however, the cutting force increased with an increase in the equivalent chip thickness. The adopted model for the specific energy in this study did not explain the material removal phases and their corresponding energy components.

In all previous studies measuring the specific energy consumption from the grinding and abrasive cutting of metal, stone, and optical glass [6,19-22], the cutting power $(P)$ is measured in terms of the tangential forces $\left(F_{\mathrm{t}}\right)$ and cutting velocity $\left(V_{\mathrm{c}}\right)$ of the disc, and shown as

$P=F_{\mathrm{t}} V_{\mathrm{c}}$.

This study aims to introduce an alternative method to directly measure the cutting power for a specific energy evaluation of an abrasive cut off operation. This methodology uses a combination of analytical methods and specially designed and manufactured equipment to automate the movement of a standard grinder at different feed rates. In addition, particularly in studies of abrasive cutting operations, the specific energy has not been distinguished into three modes during material removal $[19,23]$. However, in this research, the specific energy measured through an alternative method is categorized into three modes, i.e., sliding, plowing, and cutting, using an empirical model. These three components used in grinding have been discussed in the literature review because grinding also involves metal cutting with abrasive discs and has been extensively discussed and analyzed. The research presented below will help understand how the material properties and cutting conditions affect the behavior of the specific energy consumption during the material removal.

\section{Materials and methodology}

\subsection{Workpiece material and cutting tool}

The materials used in this experiment were S235JR, C45K, and ferrous aluminum-based intermetallic alloy $\mathrm{FeAl}$ (40\%) with a chemical composition of $60 \% \mathrm{Fe}$ and $40 \% \mathrm{Al}$ [24]. C45K was studied in three different states: normal $\mathrm{C} 45 \mathrm{~K}$ condition, $\mathrm{C} 45 \mathrm{~K}$ with quenching (C45K-Q), and $\mathrm{C} 45 \mathrm{~K}$ with quenching and tempering $(\mathrm{C} 45 \mathrm{~K}-\mathrm{Q}+\mathrm{T})$. The chemical compositions of S235JR and $\mathrm{C} 45 \mathrm{~K}$ are listed in Table $1[25,26]$. 
Table 1 Chemical composition of metallic alloys

\begin{tabular}{llllllll}
\hline Metallic alloys & $\mathrm{C}$ & $\mathrm{Mn}$ & $\mathrm{P}$ & $\mathrm{S}$ & $\mathrm{Si}$ & $\mathrm{Ni}$ & $\mathrm{Cr}$ \\
\hline C45K & 0.5 & $0.50-0.90$ & 0.03 & 0.04 & 0.40 & 0.40 & 0.40 \\
S235JR & 0.22 & 1.60 & 0.05 & 0.05 & 0.05 & & \\
\hline
\end{tabular}

The hardness of materials (HRB) given in Table 2 is measured using a Rockwell hardness tester (developed by Wilson) with an indenter load of $100 \mathrm{~kg}$. The abrasive cutting action is conducted using an ultra-thin steel cutting disc with a $1 \mathrm{~mm}$ thickness, $22 \mathrm{~mm}$ bore diameter, and 115 $\mathrm{mm}$ outer diameter mounted on a $600 \mathrm{~W}$ Ryobi grinder. The technical characteristics of the cutting disc are defined by the code 41 99A 46 S7 BF given in the catalog [27]. The first digit code 41 indicates the manufacturer symbol; 99A indicates that the abrasive material is made of aluminum oxide; 46 indicates a medium hard grain size; S7 is the hardness; and $\mathrm{BF}$ represents a type of resin used for bonding. The abrasive material of the cut off disc with a coarse grain size and high hardness enables a fast cutting $[28,29]$.

\subsection{Methodology}

The specific energy consumption is the power required to remove the unit volume of material, and its value $\left(V_{\mathrm{SEC}}\right)$ is defined as the ratio between the power consumed and the material removal rate, and shown as [6]

$V_{\mathrm{SEC}}=\frac{P_{\mathrm{m}}}{Q_{\mathrm{w}}}$,

where $P_{\mathrm{m}}$ is the mechanical cutting power, and $Q$ w is the material removal rate. To measure the mechanical cutting power, two different experimental settings were applied during the process, as shown in Fig. 1. In the first setting, as shown in Fig. 1a, the standard angle grinder was coupled with a dynamometer to measure and determine the relationship between the mechanical power and electrical power consumed under different loading conditions. The Hysteresis Dynamometer, model HD-710-BNA, developed by Magtrol, USA, was used for this experiment. A highspeed programmable controller attached to the

Table 2 Material hardness

\begin{tabular}{lr}
\hline Metallic alloys & \multicolumn{1}{c}{ HRB } \\
\hline C45K & 100.20 \\
C45K-Q & 120.60 \\
C45K-Q+T & 85.85 \\
S235JR & 80.05 \\
Intermetallic & 100.20 \\
\hline
\end{tabular}

dynamometer displays the measurement of mechanical characteristics such as torque, mechanical power $\left(P_{\mathrm{m}}\right)$, speed of rotation $(\mathrm{r} / \mathrm{min})$, and electrical characteristics such as the effective electric power $\left(E_{\mathrm{e}}\right)$ and voltage.

The average or effective electric power $E_{\mathrm{e}}$ is the amount capable of transforming the electrical energy into work. Part of the dynamometer equipment measures the effective electric power. This device does this by multiplying the current and voltage instantaneously using a 4-quadrant multiplier, and then averages the instantaneous power signal. The mechanical power $\left(P_{\mathrm{m}}\right)$ is defined as the product of the torque and angular velocity. The relationship between the mechanical power $\left(P_{\mathrm{m}}\right)$ delivered by the grinder and the effective electric power $\left(E_{\mathrm{e}}\right)$ consumed by the motor is shown in Fig. 2.

$P_{\mathrm{m}}=2.4 E_{\mathrm{e}}-742.61$

The behavior of this regression curve is associated with the grinder used and is therefore an experimental result of the behavior of the equipment used. The trend line between these two parameters is linear. Equation (3) is a regression equation of this trend line, and 2.4 denotes the slope of the line. Equation (3) obtained through this graph is particular to this grinder and can be used to determine the mechanical power during metal cutting with an abrasive disc for repetitive experiments. Using the regression equation to measure the mechanical power through the electrical energy consumed during abrasive cutting with a disc eliminates the need to measure the cutting force and cutting velocity for specific energy estimation. In the second step, a standard angle grinder is mounted on the experimental configuration, which has been specifically designed and manufactured to mount and operate the standard disc grinder at various feed rates for the metal abrasive cutting operation studied herein. Figure $1 \mathrm{~b}$ illustrates the different parts of the equipment developed for this study. An ultrathin disc of $1 \mathrm{~mm}$, mounted on the angle grinder, acted as a cutting tool to machine the metallic bars. The stepper motor operates the machine at four different feed rates defined by the potentiometer. The LED lights were used as indicators of four defined speeds. Two vertical separate buttons were placed for the clockwise and anticlockwise movement of the stepper motor, which eventually moved the machine up and down.

A schematic diagram of the electrical components inside a wooden box is shown in Fig. 3. Owing to machine requirements for higher accuracy in movement, positioning, and varying torque in dynamic loading, a stepper motor was selected [30]. The speed, movement, and direction of the stepper motor in relation to the input feed rate were programmed using the Arduino Uno microcontroller. The stepper motor drive TB6600 controls the current between the stepper motor and control circuit in micro- 


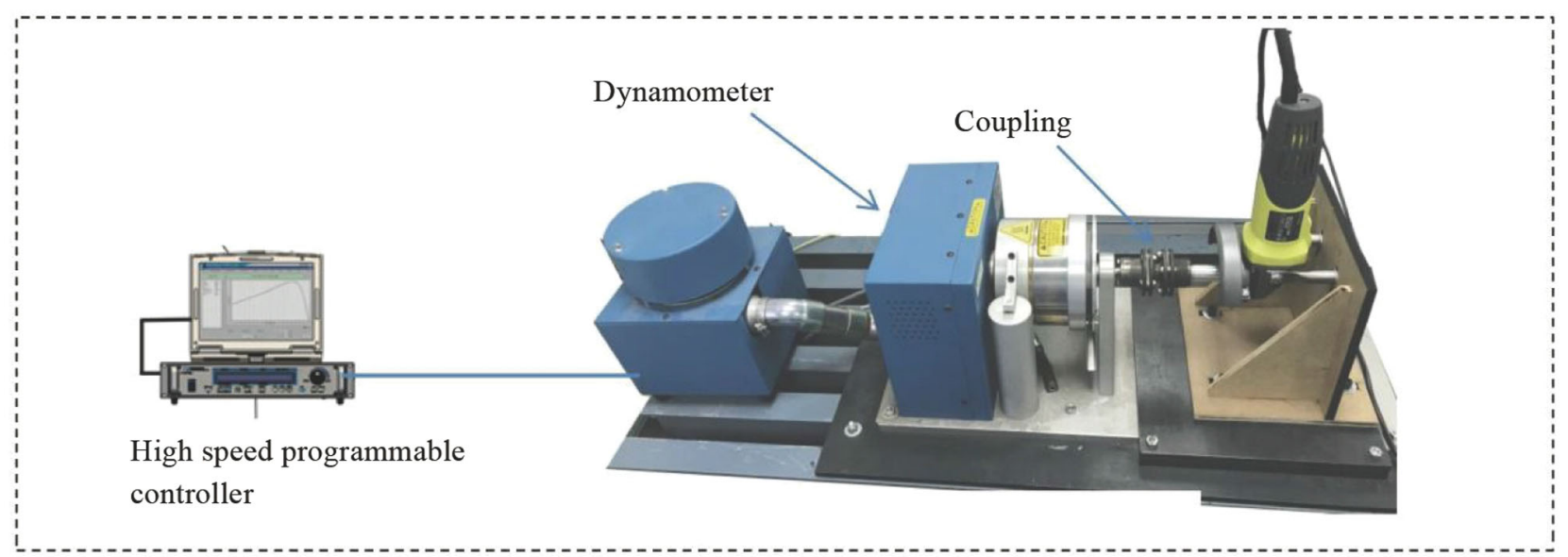

(a) Angle grinder coupled with dynamometer to measure torque

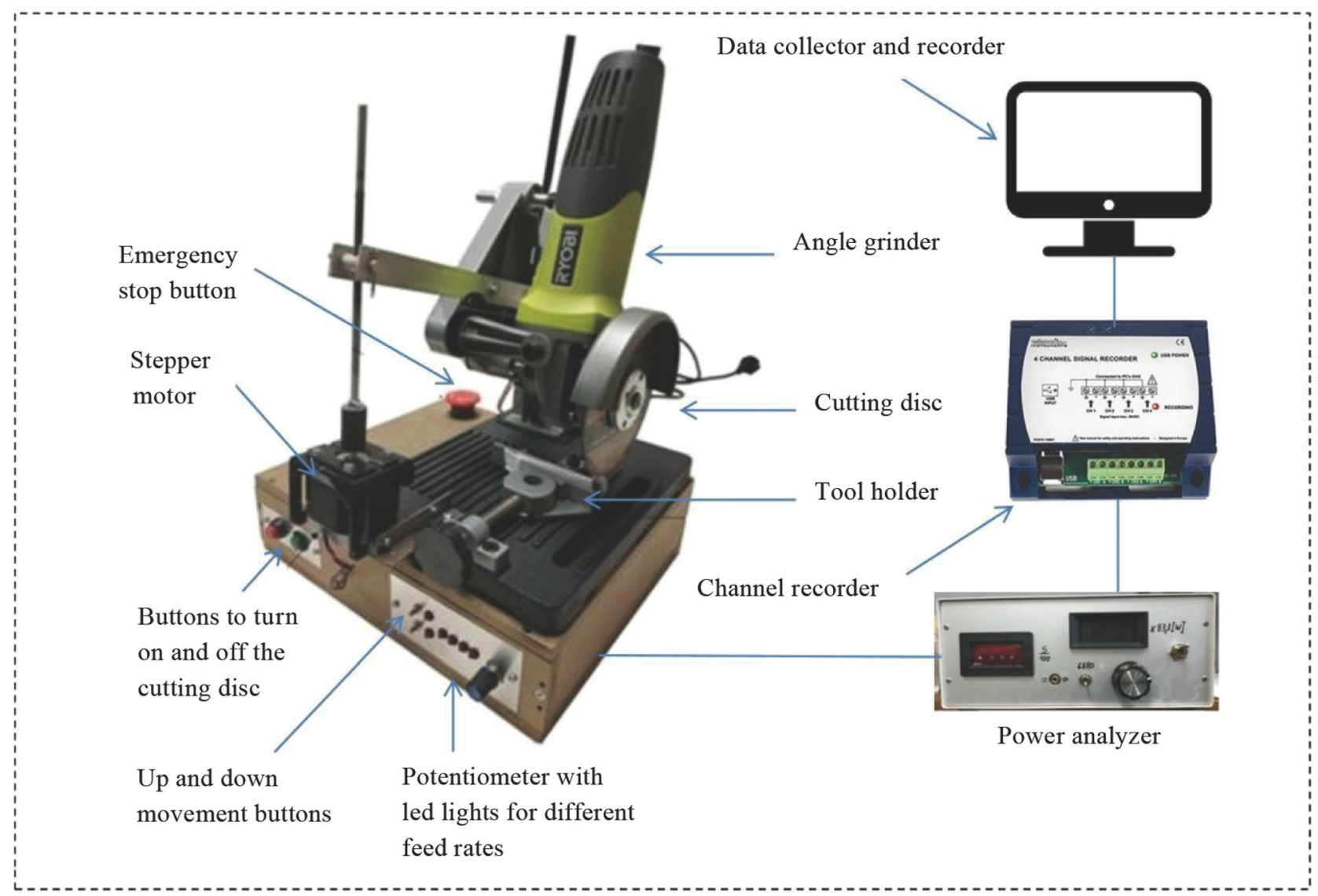

(b) Second experimental setting to measure the power consumed during the process

Fig.1 Experimental set up to measure the mechanical power and specific energy consumption

steps. The feed rate range can be changed by changing the number of steps in the drive. Grinder buttons, emergency stop buttons, and limit switches are controlled through a switch relay. Limit switches are programmed with a microcontroller, which stops the stepper motor once the movement reaches the maximum upward or minimum downward limit on the lead screw. The power source is used to step the input voltage supply down to $12 \mathrm{~V}$ for the stepper motor drive and other functions.

The electrical energy consumed by the machine during the cutting off operation is measured using a power analyzer at different feed rates, which is stored in a computer through a channel recorder. Using these electrical energy 


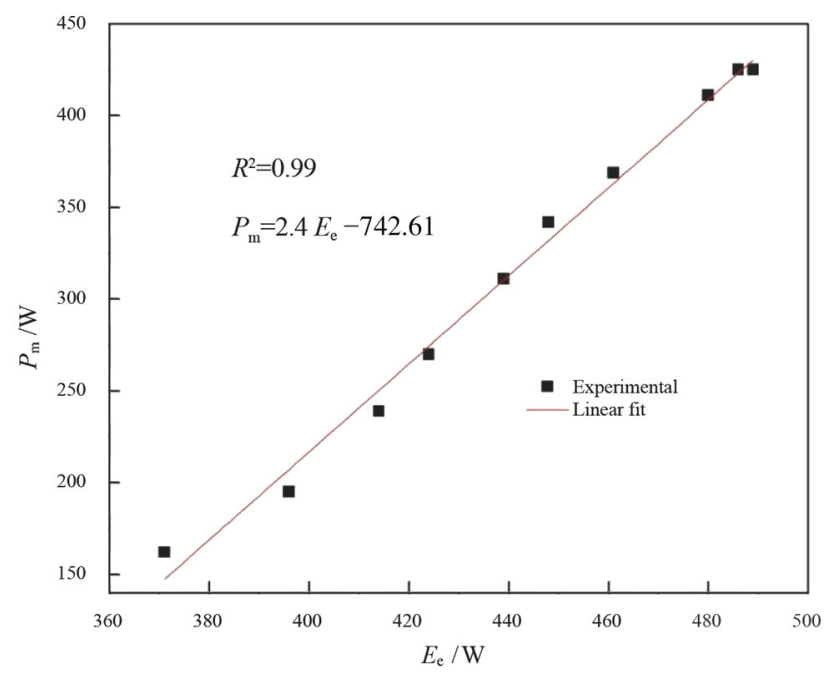

Fig. 2 Mechanical power versus electrical energy

values, the corresponding values of the mechanical power are calculated using the regression line in Eq. (3).

To measure the material removal rate in Eq. (2), a general model of the material removal rate for this experiment has been used, which is the product of the feed rate and cutting cross section [31], and shown as

$Q_{\mathrm{w}}=A_{\mathrm{c}} V_{\mathrm{f}}$,

where $V_{\mathrm{f}}$ is the feed rate, and $A_{\mathrm{c}}$ is the cutting cross-section. $V_{\mathrm{f}}$ in Eq. (4) is a function of the rotational speed of the stepper motor. A kinematic study of the spatial movement of the center point, where the cutting disc was mounted, was carried out using generalized coordinates [32]. The kinematic study showed that given a constant rotation speed of the stepper motor, the feed rate at the cutting point was vertical and remained constant throughout the cutting cross section. The feed rate values obtained from the kinematic studies were verified by the time taken for the cutting discs to cut through the length of the workpiece. The cutting cross-section $A_{\mathrm{c}}$, shown in Eq. (4), is perpendicular to the feed rate, and is defined by the product of

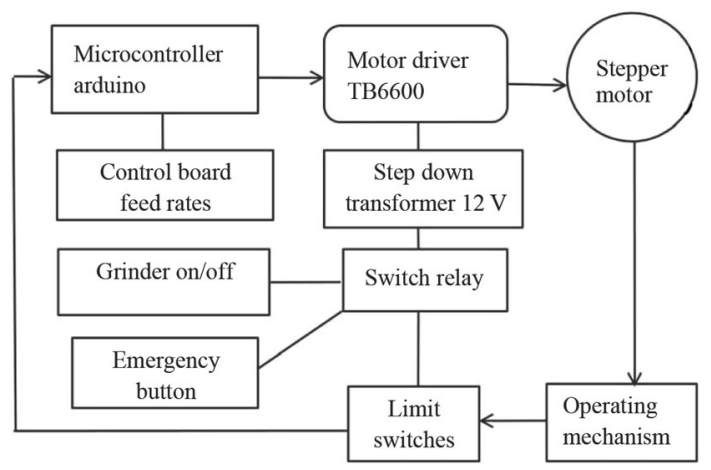

Fig. 3 Schematic diagram of electrical instruments material thickness " $b$ " and width of cutting thickness " $a$ ", as shown in Fig. 4. The widths of the specimens were 3, 10 and $4.6 \mathrm{~mm}$ for S235JR, C45K, and intermetallic $\mathrm{Fe}-\mathrm{Al}$ (40\%), respectively, and the cutting thickness was $1.8 \mathrm{~mm}$. The cutting thickness was found to be higher than that of the cutting disc. It was verified that the actual thickness of the cutting did not coincide with the information provided by the manufacturer for a $1 \mathrm{~mm}$ thick cutting disc. It was also determined that an axial deviation of the cutting disc occurred when rotated under free cutting conditions. The feed rates used in this experiment were high for these types of discs. Therefore, during the material cutting, a further bending and vibration of the cutting disc increased the cutting thickness.

\subsection{Measurement of experimental data}

The electrical energy consumed during the process was measured with an analog power analyzer built with an AD633 chip, which provided the instantaneous product values of the current and voltage. A channel recorder logger Velleman K8047 connected to a computer allows collecting the power signal with a sampling frequency of $0.01 \mathrm{~s}$. Figure 5 shows the power curve for a cutting experiment on S235JR material with $V_{\mathrm{f}}$ at $1.488 \mathrm{~mm} / \mathrm{s}$.

The behavior observed during all experiments was similar and could be divided into three stages. The first stage corresponds to the energy consumption when the disc is not cutting. The second stage is the transition stage in which cutting begins, characterized by a rapid increase in the cutting power. The third stage is the stabilization stage of the power consumption. The average power consumed during the last stage, $E_{\mathrm{e}}$, is used to estimate the specific energy consumption. Using the regression in Eq. (3), $P_{\mathrm{m}}$ is obtained as a function of the measured $E_{\mathrm{e}}$. The cutting thickness of each specimen was measured using a WADEO iT33-MDUK digital microscope with a 2 Mega pixel image sensor. The thickness was measured using Image J2 opensource software for a scientific image analysis, developed by the University of Wisconsin-Madison. The width of the specimen was measured with a digital vernier caliper. The

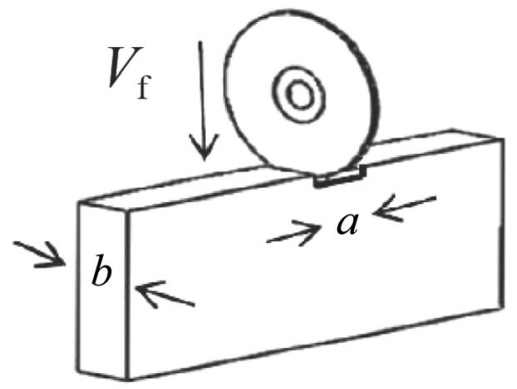

Fig. 4 Cutting cross section 
equipment allowed the selection of four different feed rates previously set at $0.539,0.613,0.899$ and $1.488 \mathrm{~mm} / \mathrm{s}$. Owing to the probability of a lack of uniformity in the grain alignment of the workpiece material and cutting disc, and a small lateral vibration of the cutting disc at lower feed rates, a small change in the material removal rate was found for the same feed rate. To compensate for this, the cutting experiment was repeated four times for each feed rate.

The sliding power $\left(P_{\mathrm{sl}}\right)$ was measured through a separate experiment, as shown in Fig. 6. The cutting disc is engaged with the workpiece material at a specific feed rate. When the cutting disc reached the middle of the cutting path and the power consumption was stabilized, the feed rate was stopped at point $\mathrm{A}$, with the cutting disc maintained in the running condition. Stopping the feed rate produced a sudden drop in power, which was again stabilized at point $\mathrm{P}$. This operation kept the cutting disc in contact with the material without chip removal and without producing a plastic deformation. The power measured at the intersection point $\mathrm{P}$ of regression lines $\mathrm{BC}$ and $\mathrm{DE}$ was only associated with the sliding phenomenon.

\subsection{Cutting model}

The cutting model is based on the fact that the mechanical power consumed by cutting $\left(P_{\mathrm{m}}\right)$ is equal to the summation of the power consumed by sliding $\left(P_{\mathrm{sl}}\right)$, power consumed by plowing $\left(P_{\mathrm{pl}}\right)$, and power consumed by the chip formation $\left(P_{\mathrm{ch}}\right)$, and shown as

$P_{\mathrm{m}}=P_{\mathrm{sl}}+P_{\mathrm{pl}}+P_{\mathrm{ch}}$.

The relationship between the specific cutting energy and material removal rate shown in Figs. 7 and 8 is estimated by the empirical model, and shown as

$\frac{P_{\mathrm{m}}-P_{\mathrm{sl}}}{Q_{\mathrm{w}}}=\frac{P_{\mathrm{pl}}}{Q_{\mathrm{w}}}+V_{\mathrm{SCE}}$.

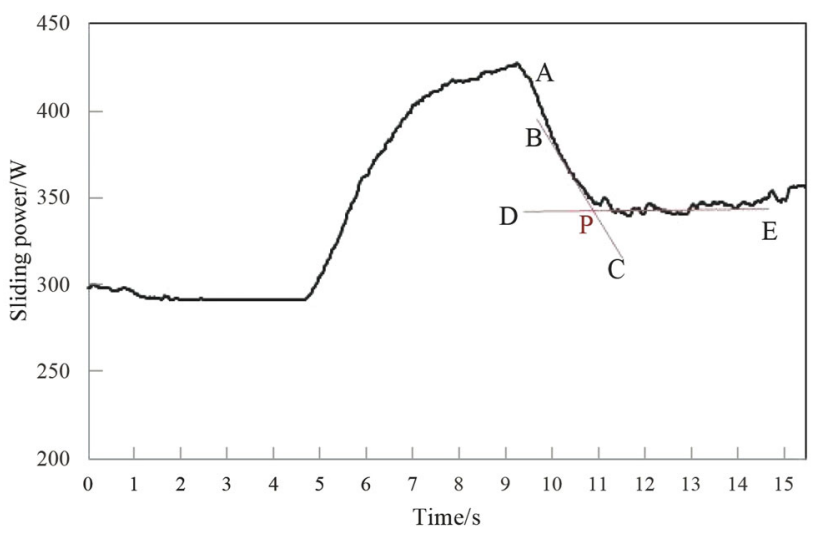

Fig. 6 Sliding power measurement for C45K-Q+T

The model presented in Eq. (6) was proposed by Gutowski et al. [33] and later validated by Kara and Li [34] based on experimental results. The adopted model demonstrated statistical accuracy in evaluating the specific energy consumption with respect to the material removal rate for the processes of grinding, milling, turning, and injection molding [35]. The experimental values of specific energy consumption for the $\mathrm{C} 45$, intermetallic $\mathrm{Fe}-\mathrm{Al}(40 \%)$, and S235JR are shown in Figs. 7 and 8, respectively. This was verified through the proposed model in Eq. (6). The powers dissipated by $P_{\mathrm{sl}}, P_{\mathrm{pl}}$ and specific cutting energy for a material are constant, as shown in Table 3 . These constant values of $P_{\mathrm{pl}}$ and specific cutting energy are the coefficients of Eq. (7) and used to draw the experimental graphs in Figs. 7 and 8. These coefficients were obtained by adjusting the experimental data using the least squares. Figures 7 and 8 indicate that the behavior of this model is asymptotic. With an increase in the material removal rate, the ratio of $P_{\mathrm{pl}}$ to $Q_{\mathrm{w}}$ decreases, and at an extremely high material removal rate, the plowing energy reduces significantly. The reduction in the plowing energy in terms of

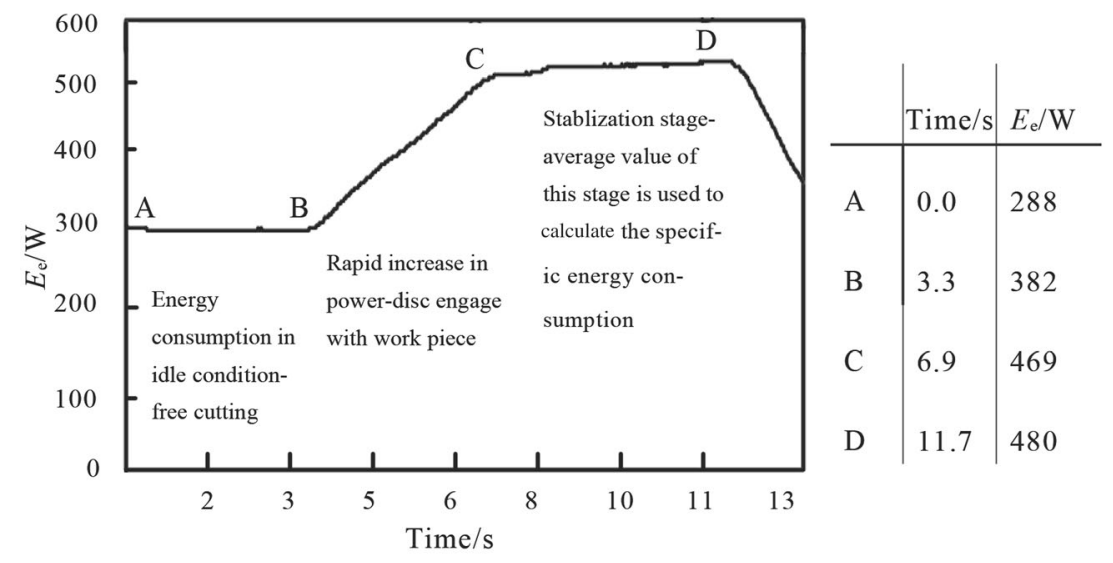

Fig. 5 Electrical energy consumption behavior 


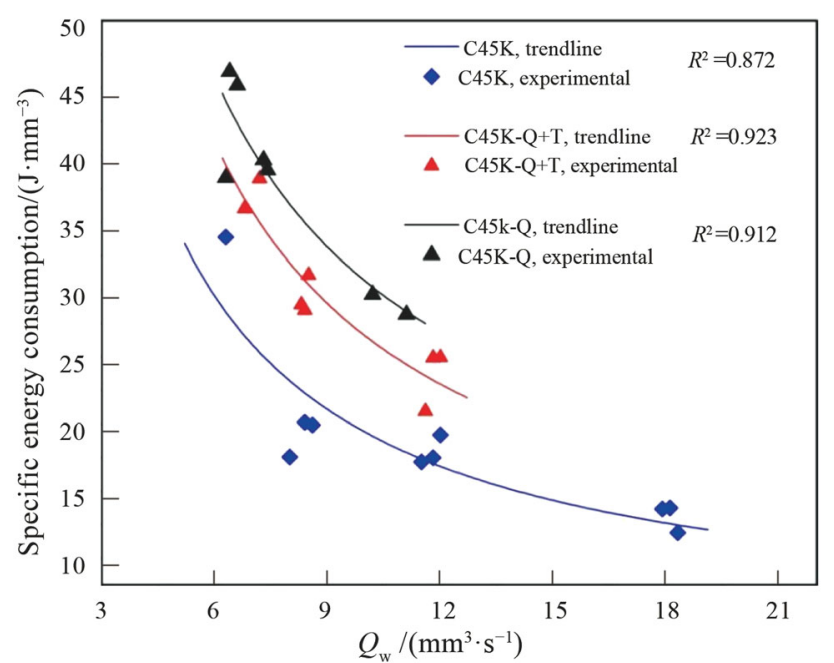

Fig. 7 Specific energy consumption relationship with material removal rate for $\mathrm{C} 45 \mathrm{~K}, \mathrm{C} 45 \mathrm{~K}-\mathrm{Q}+\mathrm{T}, \mathrm{C} 45 \mathrm{~K}-\mathrm{Q}$

percentage is also shown in Fig. 10. The reduction of plowing energy with the material removal rate is in agreement with previous research on grinding $[6,14]$. The sliding power depends on the rotational speed of the cutting disc. In this experiment, the rotational speed of the cutting disc is constant and undergoes an extremely small change depending on the feed rate. Therefore, the sliding power remains constant. The regression applied to the experimental data of the materials has coefficients of regression $R^{2}$ greater than or equal to 0.872 . The coefficient of determination, close to unity for most of the materials, shows a good fit between Eq. (6) and the experimental data of the specific energy consumption.

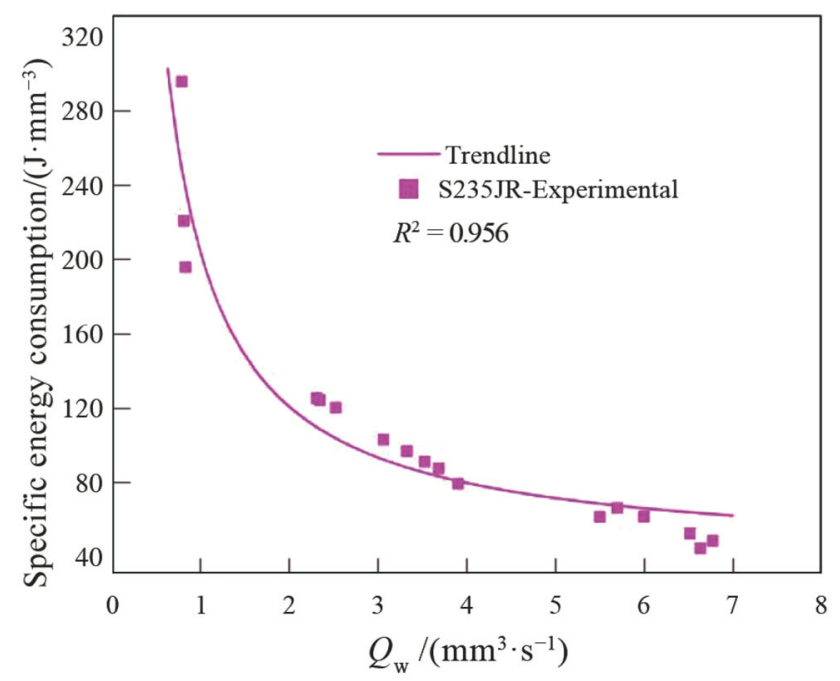

(a)
Table 3 Asymptotic parameter values and sliding power

\begin{tabular}{lcclc}
\hline Materials & $\begin{array}{l}P_{\mathrm{sl}} l \\
\left(\mathrm{~J} \cdot \mathrm{s}^{-1}\right)\end{array}$ & $\begin{array}{l}P_{\mathrm{pl}} / \\
\left(\mathrm{J} \cdot \mathrm{s}^{-1}\right)\end{array}$ & $\begin{array}{l}V_{\mathrm{SEC}} / \\
\left(\mathrm{J} \cdot \mathrm{mm}^{-3}\right)\end{array}$ & $R^{2}$ \\
\hline S235JR & 29.16 & 163.69 & 37.50 & 0.956 \\
$\begin{array}{l}\text { Intermetallic- } \\
\text { FeAl(40\%) }\end{array}$ & 77.36 & 113.19 & 13.08 & 0.878 \\
C45K-Q & & & & \\
C45K-Q+T & 99.36 & 228.04 & 7.82 & 0.912 \\
C45K & 117.36 & 216.21 & 4.93 & 0.923 \\
\hline
\end{tabular}

\section{Results and discussion}

A single factor one-way analysis of variance (ANOVA) was applied to the mean specific energy values for the four feed rates, as shown in Table 4. The variations in the mean values of the specific energy consumption were statistically significant ( $P$ values of less than 0.05 ) for most of the materials. Therefore, the feed rates used to produce different energy consumptions do not belong to the same family of cutting conditions.

In general, for all materials, the specific energy consumption decreases asymptotically with an increase in the material removal rate, which confirms the accuracy of the adopted methodology. It is evident from the graphs in Figs. 7 and 8 that, at an extremely small material removal rate, the specific energy consumption is quite high. This phenomenon is known as the size effect [6]. With an increase in the material removal rate, the specific energy consumption decreases. Because the specific energy consumption for a material deformation has a constant value,

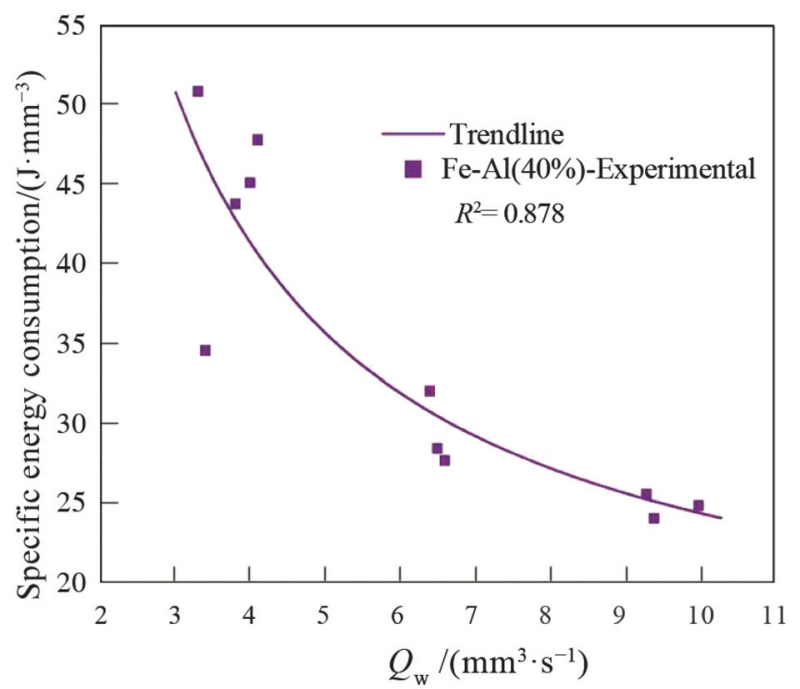

(b)

Fig. 8 Specific energy consumption relationship with material removal rate for a S235JR and b intermetallic $\mathrm{Fe}-\mathrm{Al}(40 \%)$ 
Table 4 One way ANOVA

\begin{tabular}{lllllll}
\hline Source & SS & df & MS & $F$ & $P$-value & $\begin{array}{l}F \text { - } \\
\text { critical }\end{array}$ \\
\hline C45K & 326.49 & 3 & 108.8 & 75.784 & $\begin{array}{r}3.68 \times \\
10^{-5}\end{array}$ & 4.757 \\
& & & & & 0.06456 & 5.143 \\
C45K- & 147.3 & 2 & 73.65 & 4.4783 & 0.001027 & 5.143 \\
Q+T & & & & & & \\
C45K-Q & 343.13 & 2 & 171.6 & 26.736 & 0.000418 & 4.347 \\
Fe- & 941.94 & 3 & 314 & 24.823 & 0.004 \\
Al(40\%) & & & & & & \\
S235JR & 80.468 & 3 & 26.823 & 57.243 & $9.49 \times$ & 3.411 \\
& & & & & $10^{-8}$ & \\
\hline
\end{tabular}

this decrease in the specific energy consumption is only due to the decrease in the plowing energy $\left(\frac{P_{\mathrm{pl}}}{Q_{\mathrm{w}}}\right)$. Thus, at a higher stock removal rate, the specific energy consumption is reduced to the minimum value.

This minimum energy is the specific cutting energy, which remains constant throughout the entire cutting process, as confirmed by Malkin and Guo [6]. According to Shaw [17], in the stock removal grinding process, most of the energy was dissipated as the shear cutting energy required to produce the chips. However, the author conducted an abrasive cutting off operation at extremely high feed rates $(5-60 \mathrm{r} / \mathrm{min}$ or $2.1-25.4 \mathrm{~mm} / \mathrm{s})$ [19]. The dominance of the shear cutting energy in their experiments was attributed to the use of extremely high feed rates. The range of feed rates during this experiment $(0.5-1.488 \mathrm{~mm} / \mathrm{s})$ is low in comparison to the feed rates used by Shaw [17]. The given feed rates are supported by the grinder used, and feed rates higher than this range stopped the cutting machine. Lower feed rates allowed the cutting disc to sufficiently deform the material before breaking, which was the reason for the dominance of the plowing energy and the low specific cutting energy.

Figure 7 shows the relationship between the specific energy consumption and the material removal rate for the three materials of normal $\mathrm{C} 45 \mathrm{~K}$ and $\mathrm{C} 45 \mathrm{~K}$ with different thermal treatments. C45K-Q shows the highest specific energy consumption with the highest hardness, followed by C45K-Q+T with the lowest hardness. C45K has the lowest specific energy consumption among the three materials, although its hardness is higher than that of C45K-Q+T. The specific energy consumption of these three materials and their correlation with the material hardness exhibit a different trend from the previous research conducted by Nápoles et al. [14] on the same materials for surface grinding. Thermal treatment significantly affected the specific energy consumption and specific energy consumption of the materials. The specific energy consumption of $\mathrm{C} 45 \mathrm{~K}-\mathrm{Q}$ steel is the highest among the three specimens of $\mathrm{C} 45 \mathrm{~K}$ owing to its high hardness. Marinescu et al. [36] reported that an increase in hardness increased the resistance to abrasive machining, thereby increasing the cutting forces and machining difficulty. C45K tempered steel consumes a higher specific energy compared with C45K despite the low hardness owing to the tempering conducted to reduce the hardness and increase the toughness and ductility of the material [37]. An increase in toughness increases the energy required to deform the material [38]. The three materials show the same trend of decrease in the specific energy consumption.

However, for quenched and tempered steel, at a high material removal rate, the machine did not provide an accurate representation of the energy consumed, and the cutting disc was jammed into the workpiece. This indicates that thermal treatment has a considerable effect on the machinability of the materials. It also shows that this cutting disc has limitations for specific materials under certain cutting conditions. Thermal treatment of $\mathrm{C} 45 \mathrm{~K}$ also affects the specific cutting energy. As shown in Table 3, C45K, C45K$\mathrm{Q}+\mathrm{T}$, and $\mathrm{C} 45 \mathrm{Q}$ exhibit considerable differences in the values of specific cutting energy. However, according to Malkin and Joseph [18], the minimum energy required to produce chips in the abrasive process is unaffected by alloying or heat treatment. Thus, the difference in the specific cutting energy owing to the heat treatment of $\mathrm{C} 45 \mathrm{~K}$ used in this experiment is not in agreement with Ref. [18].

Among the metallic alloys, S235JR, which has the lowest hardness, had the highest specific energy consumption and specific cutting energy, as shown in Fig. 8a. The carbon content of S235JR is comparatively lower among specimens, and low carbon steel is more ductile. Thus more energy is required to machine the low-carbon steel in comparison to the medium-carbon steel and is comparatively difficult to machine [39]. In comparison to brittle materials, ductile materials undergo extensive plastic deformation before fracture [40]. A large amount of plastic deformation in ductile materials requires a high specific energy to fracture the material [41, 42]. Therefore, the high values of specific energy consumption and specific energy consumption for S235JR are attributed to a large plastic deformation owing to a high ductility.

The specific energy consumption behavior of the intermetallic $\mathrm{Fe}-\mathrm{Al}(40 \%)$ is shown in Fig. 8b. The hardness of metallic $\mathrm{Fe}-\mathrm{Al}(40 \%)$ is the same as that of $\mathrm{C} 45 \mathrm{~K}$, and its specific energy consumption relationship with material removal follows the same trend as that of the normal C45K. C45K is brittle owing to its higher carbon content. The same trend of expansion is attributed to the brittle nature of the intermetallic $\mathrm{Fe}-\mathrm{Al}(40 \%)$. The brittle fracture of intermetallic materials is due to weak grain boundaries and the higher concentration of aluminum, which limits the ductility of the material [24, 43, 44]. However, the specific 
energy consumption of intermetallic $\mathrm{Fe}-\mathrm{Al}(40 \%)$ is higher than that of the $\mathrm{C} 45 \mathrm{~K}$ normal and with thermal treatment. As shown in Table 3, the specific cutting energy of intermetallic $\mathrm{Fe}-\mathrm{Al}(40 \%)$ in percentage of total energy is higher than that of normal $\mathrm{C} 45 \mathrm{~K}$ and with thermal treatment. This indicates the comparatively slightly higher ductility of intermetallic $\mathrm{Fe}-\mathrm{Al}(40 \%)$, which is the reason for the high specific cutting energy. Saigal and Yang [45] also reported higher cutting forces for iron aluminides in comparison to medium-carbon steel for milling operations.

Figure 9 shows a comparison of the sliding power per unit of cutting area for all materials. The experiment results in Fig. 6 show that the sliding energy does not participate in the material removal process and is merely a rubbing or friction energy. For this reason, sliding power per unit of cutting area was used by Zhang et al. [46] and Linke et al. [47]. The highest value of the sliding power per unit cutting area for intermetallic $\mathrm{Fe}-\mathrm{Al}(40 \%)$ was due to its brittle nature at normal temperatures. The sliding or rubbing power is dissipated during the initial contact of the cutting grain with the material, and does not produce a material deformation. Brittle materials do not undergo any plastic deformation before a fracture [31]. Therefore, the high sliding power for intermetallic $\mathrm{Fe}-\mathrm{Al}(40 \%)$ is due to the initial rubbing energy being absorbed by the material without producing plastic deformation. Wu et al. [48] presented a model that also provided some support to this argument, which stated that, in the grinding of brittle materials, the main forms of energy dissipation were rubbing and chipping. S235JR has the highest ductility among the given materials, and ductile materials tend to undergo significant plastic deformation prior to a fracture [40]. Therefore, the plastic deformation of S235JR starts extremely early, which is the reason for the very low sliding power dissipation per unit cutting area. The C45K family has a lower ductility than S235JR owing to its higher carbon content, which is the reason for the comparatively higher sliding energy per unit of the cutting area. Within the $\mathrm{C} 45 \mathrm{~K}$ family, C45K-Q has the highest hardness, which increases the brittleness, and thus, the sliding power per unit of the cutting area increases. C45K-Q+T has less sliding power per unit of cutting area than $\mathrm{C} 45 \mathrm{~K}$ because of the thermal treatment. As the tempering reduces the hardness and increases the ductility of the material, the sliding power per unit of cutting area is reduced.

To determine the percentage of contribution of the specific energy components for material removal, the bar chart shown in Fig. 10 was drawn. The sliding energy is not shown in this graph because it merely rubs and does not deform the material. For all materials, in general, the specific plowing energy has been found to be the most significant phenomenon in the total specific energy consumption. The dominance of the plowing energy for these

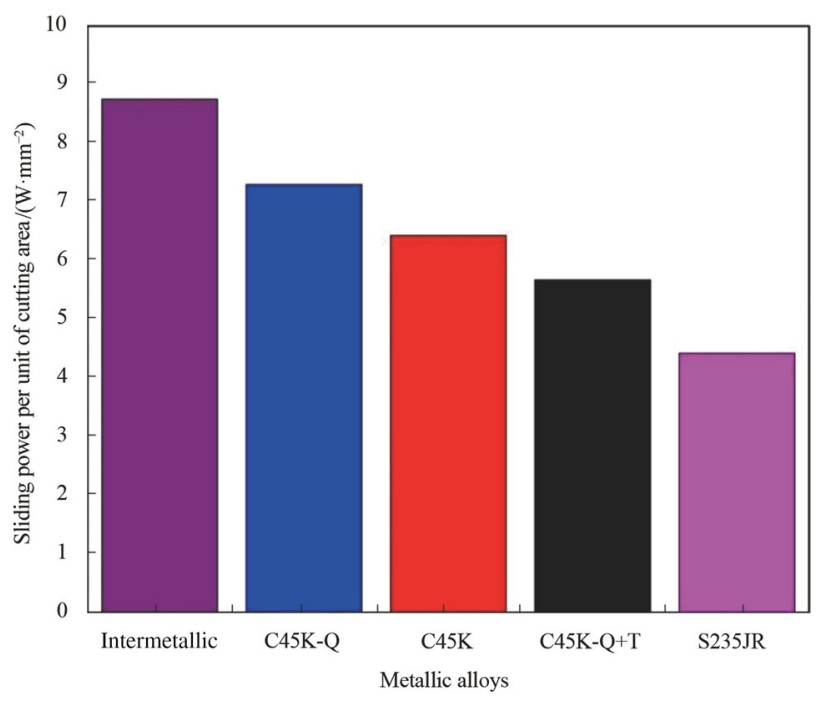

Fig. 9 Comparison of sliding power per unit of cutting area for all materials

materials is due to the use of a comparatively low feed rate for this experiment. The contribution of the plowing energy in terms of the percentage is higher for the C45 K family of materials because of their high hardness values. Even at high feed rates, plowing energy remained the most dominant phenomenon, with $68 \%, 79 \%$, and $73 \%$ for $\mathrm{C} 45 \mathrm{~K}$, $\mathrm{C} 45 \mathrm{~K}-\mathrm{Q}+\mathrm{T}$, and $\mathrm{C} 45 \mathrm{~K}-\mathrm{Q}$, respectively. The dominance of the plowing energy in hard and high-strength materials has also been determined for grinding [16, 49]. The contribution of specific energy consumption in terms of percentage was comparatively higher for S235JR at higher feed rates. Owing to the low hardness value of S235JR, the transition from elastic to plastic deformation becomes easier at higher feed rates, which rapidly reduces the plowing energy.

The cutting power depends on the material properties and feed rate. The relationships between the cutting power and the feed rates for $\mathrm{C} 45 \mathrm{~K}$ and S235JR are shown in Fig. 11. As indicated in Fig.11, the cutting power increases with an increase in the feed rate; however, at a certain higher feed rate, the increase in cutting power reduces and the curve tends to flatten. At high feed rates, the cutting grains become sharper and consume less power for metal cutting; hence, the cutting force decreases. This behavior is analogous to the research findings of Shaw [19] on abrasive cut off operations. The cutting power relationship with the feed rate for intermetallic $\mathrm{Fe}-\mathrm{Al}(40 \%)$ did not follow the general trend. As shown in Fig. 11, the cutting power at a high feed rate did not decrease and increased to a higher value. This increase in cutting power is attributed to an increase in the ductility of the material owing to the high temperature encountered at high feed rates. At high feed rates, an increase in temperature increases the yield stress; hence, the ductility of intermetallic $\mathrm{Fe}-\mathrm{Al}(40 \%)$ increases 


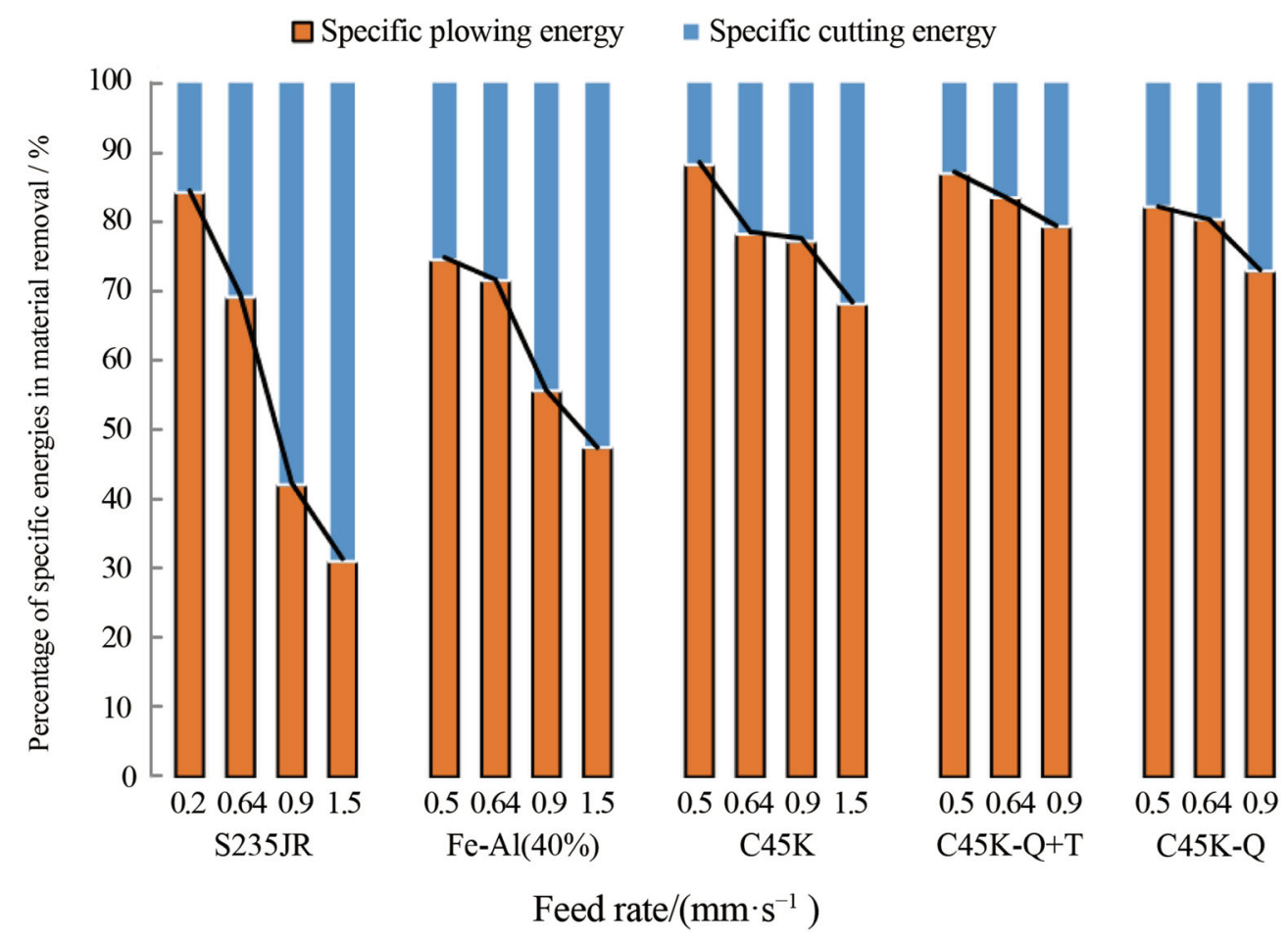

Fig.10 Percentage contribution of specific energies at different feed rates

$[50,51]$. An increase in ductility also increases the power required to cut the material.

\section{Conclusions}

An experimental setup was successfully designed and manufactured to perform the abrasive cut off operation using a commercial standard grinder and standard cutting

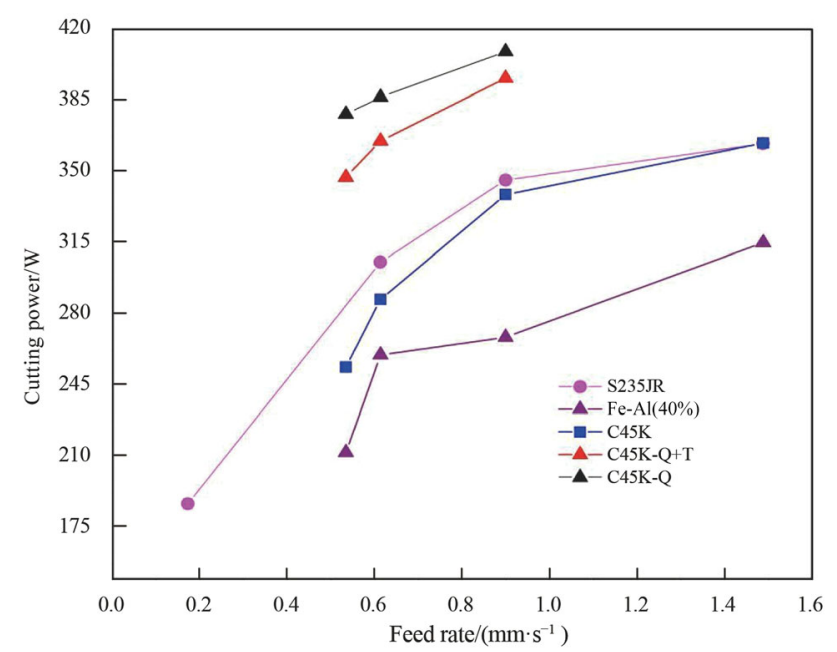

Fig. 11 Cutting power relationship with feed rate for $\mathrm{C} 45 \mathrm{~K}, \mathrm{C} 45 \mathrm{~K}$ $\mathrm{Q}+\mathrm{T}, \mathrm{C} 45 \mathrm{Q}, \mathrm{S} 235 \mathrm{JR}$ and intermetallic-FeAl(40\%) discs. The adopted experimental technique made use of conventional power measurements through a dynamometer to devise a new method of measuring the specific energy consumption. The experimental procedure was simplified using a regression equation to determine the mechanical power by simply measuring the electrical energy during abrasive cutting. The specific energy consumption was then characterized and analyzed with the change in the material removal rate. It was found that abrasive cutting with this experimental setup was suitable for comparatively brittle materials at low and medium feed rates. The specific energy consumption is the minimum amount of energy required to deform the material, and at extremely higher feed rates is the only apparent form of energy as the plowing energy becomes negligible. The material properties and thermal treatment have a significant influence on the specific energy consumption and specific cutting energy. Steel with a low carbon content has a high specific energy consumption owing to its high ductility. As the carbon content of the steel increases, the material brittleness also increases, which decreases the specific energy consumption. In the case of C45 steel, it was found that the quenching of the material increased the specific energy consumption and specific cutting energy, whereas tempering after quenching decreased the specific cutting energy and specific energy consumption. The sliding power per unit of the cutting area is higher for brittle materials and lower for materials with a comparatively higher 
ductility. Plowing energy is the most prominent phenomenon of energy dissipation for all materials. At higher feed rates, the major dominance of the specific cutting energy in terms of the percentage of the total material removal energy was observed for S235JR and intermetallic $\mathrm{Fe}-\mathrm{Al}(40 \%)$ at $69 \%$ and $53 \%$, respectively. For the $\mathrm{C} 45 \mathrm{~K}$ family of materials, even at higher feed rates, the plowing energy remained the most significant phenomenon.

Open Access This article is licensed under a Creative Commons Attribution 4.0 International License, which permits use, sharing, adaptation, distribution and reproduction in any medium or format, as long as you give appropriate credit to the original author(s) and the source, provide a link to the Creative Commons licence, and indicate if changes were made. The images or other third party material in this article are included in the article's Creative Commons licence, unless indicated otherwise in a credit line to the material. If material is not included in the article's Creative Commons licence and your intended use is not permitted by statutory regulation or exceeds the permitted use, you will need to obtain permission directly from the copyright holder. To view a copy of this licence, visit http://creativecommons. org/licenses/by/4.0/.

Funding Open Access funding provided thanks to the CRUE-CSIC agreement with Springer Nature.

\section{References}

1. IEO (2018) International Energy Outlook 2018 ( IEO2018 ) Key takeaways. In: U.S. Energy Inf. Adm. https://www.eia.gov/press room/presentations/capuano_07242018.pdf. Accessed 2 Jan, 2021

2. Rahimifard S, Seow Y, Childs T (2010) Minimising embodied product energy to support energy efficient manufacturing. CIRP Ann Manuf Technol 59(1):25-28

3. Yuan C, Zhai Q, Dornfeld D (2012) A three dimensional system approach for environmentally sustainable manufacturing. CIRP Ann Manuf Technol 61:39-42

4. Zhang YJ (2014) Energy efficiency techniques in machining process: a review. Int J Adv Manuf Technol 71(5/8):1123-1132

5. He Y, Liu F, Wu T et al (2012) Analysis and estimation of energy consumption for numerical control machining. Proc Inst Mech Eng Part B J Eng Manuf 226:255-266

6. Malkin S, Guo C (2008) Grinding technology: theory and applications of machining with abrasives. Industrial Press, New York

7. Ding H, Guo D, Cheng K et al (2014) An investigation on quantitative analysis of energy consumption and carbon footprint in the grinding process. Proc Inst Mech Eng Part B J Eng Manuf 228:950-956

8. Komanduri R, Iyengar S (2001) Conventional and super abrasive materials. In: encyclopedia of materials: science and technology, 2nd ed. Pergamon, pp 1629-1651

9. Nelson JA, Westrich RM (1974) Abrasive cutting in metallography. In: Metallographic specimen preparation. Springer, US, pp 41-54

10. Kannappan S, Malkin S (1972) Effects of grain size and operating parameters on the mechanics of grinding. J Manuf Sci Eng 94(3):833-842

11. Öpöz TT, Chen X (2012) Experimental investigation of material removal mechanism in single grit grinding. Int $\mathrm{J}$ Mach Tools Manuf 63:32-40
12. Stachowiak GW, Batchelor, Andrew WGB (2004) Experimental methods in tribology: Introduction. Tribol Ser 44:1-12

13. Rowe WB (2018) Towards high productivity in precision grinding. Inventions 3(2):24. https://doi.org/10.3390/ inventions 3020024

14. Nápoles AA, González RH, Sánchez EA et al (2019) Model based on an effective material-removal rate to evaluate specific energy consumption in grinding. Materials 12:939. https://doi. org/10.3390/ma12060939

15. Masoumi H, Safavi SM, Salehi M (2014) Grinding force, specific energy and material removal mechanism in grinding of HVOFsprayed WC-Co-Cr coating. Mater Manuf Process 29:321-330

16. Singh V, Venkateswara RP, Ghosh S (2012) Development of specific grinding energy model. Int J Mach Tools Manuf 60:1-13

17. Shaw MC (1996) Energy conversion in cutting and grinding. CIRP Ann Manuf Technol 45:101-104

18. Malkin S, Joseph N (1975) Minimum energy in abrasive processes. Wear 32:15-23

19. Shaw MC, Farmer DA, Nakayama K (1967) Mechanics of the abrasive cutoff operation. J Manuf Sci Eng Trans ASME 89(3):495-502

20. Turchetta $S$ (2010) Cutting force in stone machining by diamond disk. Adv Mater Sci Eng 2010:631437. https://doi.org/10.1155/ 2010/631437

21. Azizi A, Mohamadyari M (2015) Modeling and analysis of grinding forces based on the single grit scratch. Int J Adv Manuf Technol 78:1223-1231

22. Brach K, Pai DM, Ratterman E et al (1988) Grinding forces and energy. J Manuf Sci Eng 110:25-31

23. Jain VK, Mote RG (2005) On the temperature and specific energy during electrodischarge diamond grinding (EDDG). Int $\mathbf{J}$ Adv Manuf Technol 26:56-67

24. Villagomez-Galindo M, Torre C, Romo-Castañeda JC et al (2016) Casting Fe-Al-based intermetallics alloyed with $\mathrm{Li}$ and Ag. J Mater Res 31:2473-2481

25. Alvi A (2018) S235JR non-alloy quality structural steel. https:// www.materialgrades.com/s235jr-non-alloy-quality-structuralsteel-2062.html. Accessed 10 May 2020

26. Iman W (2018) C45 medium carbon steel grade. Jul 2018. https:// www.materialgrades.com/c45-medium-carbon-steel-grade-2082. html. Accessed 10 May 2020

27. 3M (2020) 3M, cut and grind wheel. https://www. 3m.com/3M/ en_US/metalworking-us/applications/cutting/. Accessed 3 Mar 2020

28. Wurm JD (1984) Machinist (AFSC 42750). Extension Course Institute, Air University

29. Sivasankar B (2008) Engineering chemistry. Tata McGrawHill New Delhi

30. Stepper motor or servomotor: Which should it be? https://www. motioncontroltips.com/stepper-motor-servomotor. Accessed 9 Jan 2021

31. Rowe WB (2014) Principles of modern grinding technology. Elsevier

32. García de Jalón J (1994) kinematic and dynamic simulation of multibody systems: the real-time challenge

33. Gutowski T, Dahmus J, Thiriez A (2006) Electrical energy requirements for manufacturing processes. In: Proceedings of the 13th CIRP international conference on life cycle engineering, 31 May - 2 June, 2006, Paris, France, pp 623-638

34. Kara S, Li W (2011) Unit process energy consumption models for material removal processes. CIRP Ann Manuf Technol 60(1):37-40

35. Li W, Winter M, Kara S et al (2012) Eco-efficiency of manufacturing processes: a grinding case. CIRP Ann Manuf Technol 61:59-62 
36. Marinescu ID, Rowe WB, Dimitrov B et al (2004) Tribology of abrasive machining processes. William Andrew Inc, New York, US

37. Verhoeven J (2007) Steel metallurgy for the non-metallurgist. ASM International, Almere

38. Larson B, Schmerr L (2003) Collaboration for nondestructive testing education-extending the reach. AIP Conf Proc 657(1):1899-1904

39. Astakhov VP, Outeiro JC (2008) Metal cutting mechanics, finite element modelling. In: Machining: fundamentals and recent advances. Springer, London, pp 1-27

40. Buehler MJ (2008) Atomistic modeling of materials failure. Springer Science \& Business Media

41. Yousefi R, Ichida Y (2000) Study on ultra-high-speed cutting of aluminum alloy: formation of welded metal on the secondary cutting edge of the tool and its effects on the quality of finished surface. Precis Eng 24:371-376

42. Hameed S, Rojas HAG, Benavides JIP et al (2018) Influence of the regime of electropulsing-assisted machining on the plastic deformation of the layer being cut. Materials 11(6):886. https:// doi.org/10.3390/ma11060886

43. Morris DG, Muñoz-Morris MA, Requejo LM (2007) Work hardening in Fe-Al alloys. Mater Sci Eng A 460:163-173

44. Liu CT, George EP, Maziasz PJ et al (1998) Recent advances in B2 iron aluminide alloys: deformation, fracture and alloy design. Mater Sci Eng A 258:84-98

45. Saigal A, Yang W (2003) Analysis of milling of iron aluminides. J Mater Process Technol 132:149-156

46. Zhang T, Jiang F, Yan L et al (2018) Research on the size effect of specific cutting energy based on numerical simulation of single grit scratching. J Manuf Sci Eng 140(7):071017. https://doi.org/ $10.1115 / 1.4039916$

47. Linke B, Garretson I, Torner FM (2017) Grinding energy modeling based on friction, plowing and shearing. J Manuf Sci Eng 139(12):121009. https://doi.org/10.1115/1.4037239

48. Wu C, Li B, Yang J et al (2016) Prediction of grinding force for brittle materials considering co-existing of ductility and brittleness. Int J Adv Manuf Technol 87:1967-1975

49. Sinha MK, Ghosh S, Paruchuri VR (2019) Modelling of specific grinding energy for Inconel 718 superalloy. Proc Inst Mech Eng Part B J Eng Manuf 233:443-460

50. Kupka M (2006) High temperature strengthening of the FeAl intermetallic phase-based alloy. Intermetallics 14:149-155

51. George EP, Baker I (1998) Thermal vacancies and the yield anomaly of FeAl. Intermetallics 6:759-763

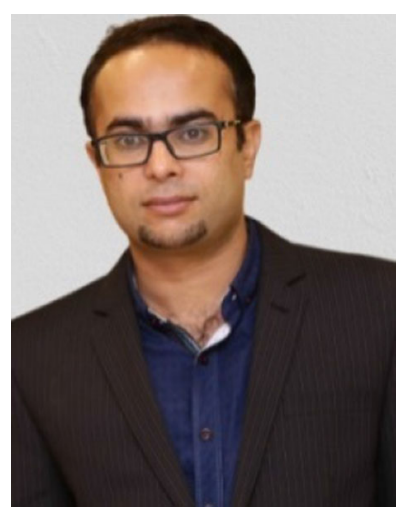

Muhammad Rizwan Awan is a $\mathrm{PhD}$ scholar of mechanical engineering in Universitat Politechnicia De CatalunyaUPC, Spain. He received his masters degree in Energy Engineering from Royal Institute of Technology-KTH, Sweden. His research interests are manufacturing processes and systems.

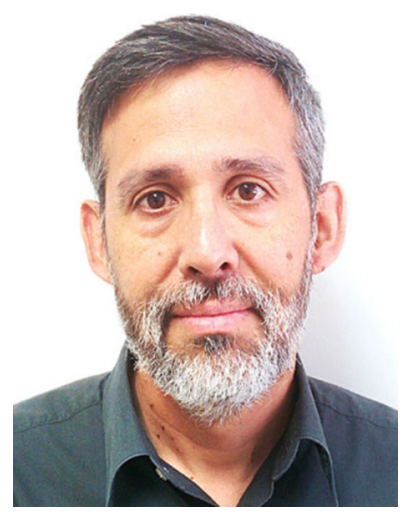

Hernán A. González Rojas is an Associate Professor in the Universitat Politècnica de Catalunya, Spain and member of the GAECE research group (Electronically Commutated Drives). He received his B.A. degree in Mechanical Engineering from Universidad de Santiago de Chile in 1993. He received his Ph.D. in Industrial Engineering from Universitat Politècnica de Catalunya in 2009. His research focuses on signal processing applied to biomedical applications and design of manufacturing systems and manufacturing processes.

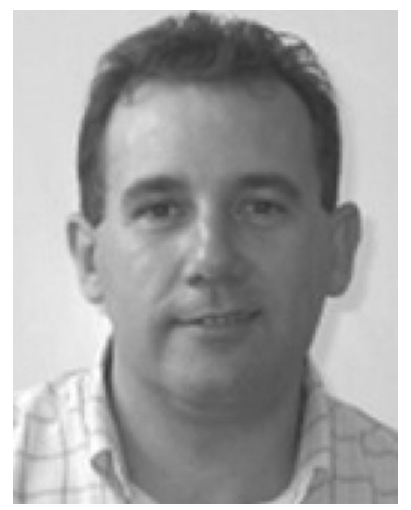

Jose I. Perat Benavides is an Associate Professor with the Department of Electrical Engineering, Polytechnic University of Catalonia. Since 1989, he has been lecturing with the School of Engineering of Vilanova i la Geltrú, where he has been a member of the GAECE Research Group (Electronically Commutated Drives). $\mathrm{He}$ is currently taking part in numerous activities related to technology transfer and lifelong learning. He has published numerous specialized articles and has participated in different national and international conferences. He is currently the Director of the Master's degree in Electric and Rail Systems.

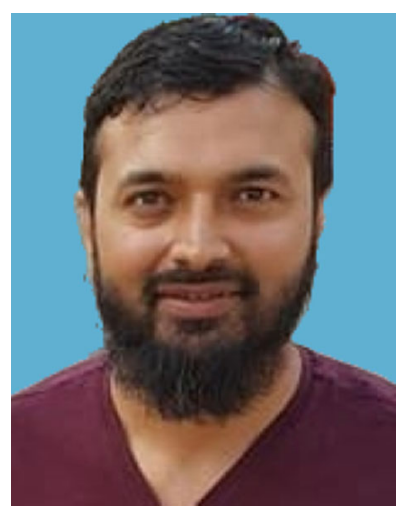

Saqib Hammed obtained his $\mathrm{PhD}$ degree in mechanical engineering from Universitat Politecnicia de Catalunya, Spain in 2017. He got his masters degree in Mechanical Engineering from Trinity College Dublin, Ireland. His research interests are manufacturing processes and systems. He has been the author and coauthor of several journal publications. 